\title{
Analysis of $T$-phase and Tsunami Signals Associated with the 2011 Tohoku Earthquake Acquired by CTBT Water-Column Hydrophone Triplets
}

\author{
Hiroyuki MATSUMOTO ${ }^{1}$, Georgios HARALABUS ${ }^{2}$, Mario ZAMPOLLI ${ }^{3}$, \\ Tomoaki YAMADA ${ }^{4}$ and Mark K. PRIOR ${ }^{5}$ \\ ${ }^{1}$ Member of JSCE, Research Scientist, Research and Development Center for Earthquake and Tsunami, JAMSTEC \\ Cost Free Expert, International Monitoring System, CTBTO \\ (Vienna International Centre, P.O. Box 1200, 1400 Vienna, Austria) \\ E-mail:hiroyuki.matsumoto@ctbto.org \\ ${ }^{2}$ Project Manager, International Monitoring System, CTBTO \\ (Vienna International Centre, P.O. Box 1200, 1400 Vienna, Austria) \\ E-mail:georgios.haralabus@ctbto.org \\ ${ }^{3}$ Hydroacoustic Engineer, International Monitoring System, CTBTO \\ (Vienna International Centre, P.O. Box 1200, 1400 Vienna, Austria) \\ E-mail:mario.zampolli@ctbto.org \\ ${ }^{4}$ Assistant Professor, Earthquake Research Institute, University of Tokyo \\ Cost Free Expert, International Monitoring System, CTBTO \\ (Vienna International Centre, P.O. Box 1200, 1400 Vienna, Austria) \\ E-mail:tomoaki.yamada@ctbto.org \\ ${ }^{5}$ Former Seismoacoustic Officer, International Data Centre, CTBTO \\ Currently, Senior Scientist, Acoustics and Sonar Department, TNO \\ (Oude Waalsdorperweg 63, 2597 AK Den Haag, Netherlands) \\ E-mail:mark.prior@tno.nl
}

\begin{abstract}
The hydrophone triplets of the Comprehensive Nuclear-Test-Ban Treaty (CTBT) International Monitoring System (IMS) hydroacoustic (HA) network have been installed in the water-column at different locations around the world's oceans since the early 2000s. The present study focuses on hydrophone data recorded at the IMS hydroacoustic station HA11 Wake Island during the 2011 Tohoku earthquake, with the purpose of estimating the directivity of $T$-phase signals and identifying tsunami warning indicators. In the first part of the paper, IMS hydrophone data not including contributions from tsunami stages are analyzed with the objective of detecting the $T$-phase and determining its direction of arrival (DOA). The second part of the study addresses the detectability of pressure variations associated with the tsunami itself. For the analysis of $T$-phase signal directivity, a three-step process is introduced to obtain a signal envelope associated with the $T$-phase which makes it possible to apply F-K analysis without spatial aliasing. It is shown that using the three-step envelope processing technique, the direction of the $T$-phase signals acquired at the IMS hydrophone triplets can be accurately estimated. For the analysis of tsunami detectability, it is shown that short period dispersive tsunami signals can be identified by IMS hydrophone triplets.
\end{abstract}

Key Words: Tohoku earthquake, hydrophone triplet, T-phase, tsunami, direction of arrival

\section{INTRODUCTION}

Bottom pressure gauges are currently the most widely used type of sensor for offshore tsunami observations. Since the 1950s, $T$-phases have been also studied in relation to tsunami warning because of their faster speed of propagation compared to tsunami waves ${ }^{11}$. Although theoretical and empirical studies regarding $T$-phases during tsunamigenic earthquakes have been previously conducted ${ }^{2-4)}$, in-situ observations based on hydrophones in the SOund Fixing And Ranging (SOFAR) channel axis are not common in the literature. This study is concerned with hydroacoustic hydrophone stations of the CTBT IMS, which have been installed in the water-column around the world since the early 


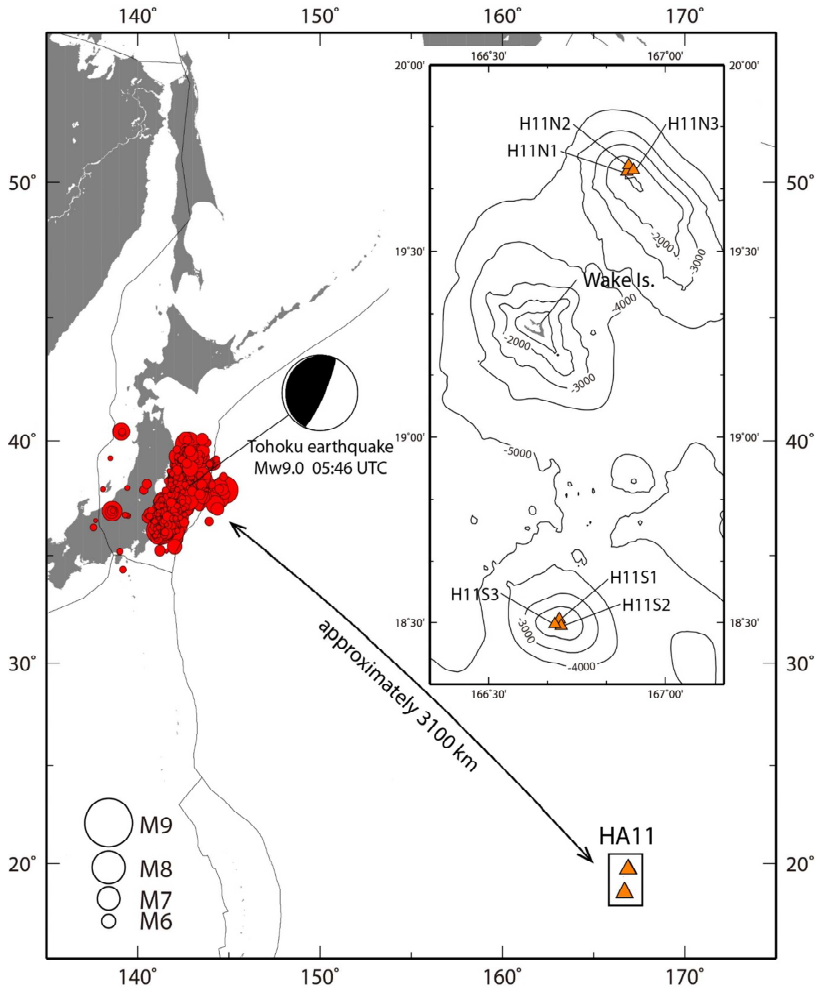

Fig.1 Map showing the 2011 Tohoku earthquake and HA11 IMS hydroacoustic station at Wake Is. The inset shows the location of the hydrophone triplets.

2000s. Further information about IMS stations is provided at http://www.ctbto.org/. Some studies using IMS hydrophones were published after the 2004 Sumatra earthquake, in which the Indian Ocean tsunami could be detected by the IMS hydroacoustic station HA08 Diego Garcia (British Indian Ocean Territory in the Central Indian Ocean) ${ }^{5,6)}$. Seismic source processing of HA08 hydrophone data from the same earthquake has been shown to be capable of identifying the propagation of the rupture by employing the Progressive Multi-Channel Correlation (PMCC) method ${ }^{7}$.

In the present study, the focus is on data recorded at the IMS hydroacoustic station HA11 Wake Island during the mega-thrust earthquake that occurred off the east coast of Japan on 11 March 2011 (hereafter referred to as the "Tohoku earthquake"). The objective is to investigate the potential use of such data for $T$-phase detection and tsunami forecasting. In order to estimate the $T$-phase DOA, array analysis is applied to the signals containing the $T$-phase arrival. The selected technique is based on frequency-wavenumber (F-K) analysis. However, in order to avoid the spatial aliasing that would result from retaining the full $100 \mathrm{~Hz}$ bandwidth data of the hydrophones, filtering and envelope waveform processing of the data is necessary. Details of this technique are described below.

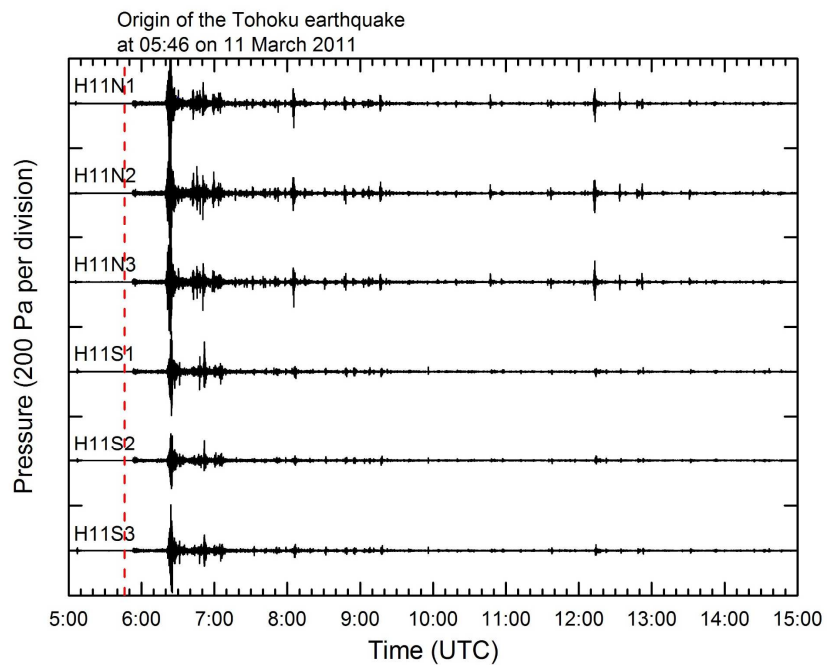

Fig.2 Pressure waveforms recorded by the HA11 hydrophone triplets. Vertical dashed line represents the origin time of the Tohoku earthquake.

\section{HYDROPHONE TRIPLET DATA FROM THE TOHOKU 2011 EARTHQUAKE}

We briefly introduce the IMS hydroacoustic station at Wake Island and the data recorded during the Tohoku earthquake. Figure 1 shows a map of the epicenter of the Tohoku earthquake and its aftershocks distribution for the following day, together with the IMS hydroacoustic station HA11 Wake Island. HA11 is located approximately $3100 \mathrm{~km}$ south-east of the epicenter of the Tohoku earthquake. HA11 consists of two hydrophone triplets; one to the North and one to the South of the island at $55 \mathrm{~km}$ and $87 \mathrm{~km}$ distance offshore respectively (inset of Figure 1). Each triplet forms an approximate equilateral triangle with sides of $2 \mathrm{~km}$. All six hydrophone sensors are suspended at a water depth of approximately $750 \mathrm{~m}$ near the SOFAR channel axis.

At the time of the Tohoku earthquake, HA11 was in operation and full waveforms between 1 and $100 \mathrm{~Hz}$ were recorded. Figure 2 shows the raw pressure waveforms for the event at each hydrophone. The top three and bottom three waveforms were recorded by the north and the south triplets respectively. The waveforms are similar between the hydrophones of each triplet. Pressure fluctuations associated with the seismic waves are recorded shortly after the earthquake. Between 06:20 and 06:30 UTC the largest waves corresponding to the $T$-phases associated with the source are recorded. The reception of strong $T$-phase arrivals is mainly attributed to the hydrophone locations near the SOFAR channel axis.

The tsunami signals are not readily recognizable in the raw data because of the low-frequency roll off of the hydrophone response, which is required in order to avoid degradation of the sensor's dynamic 


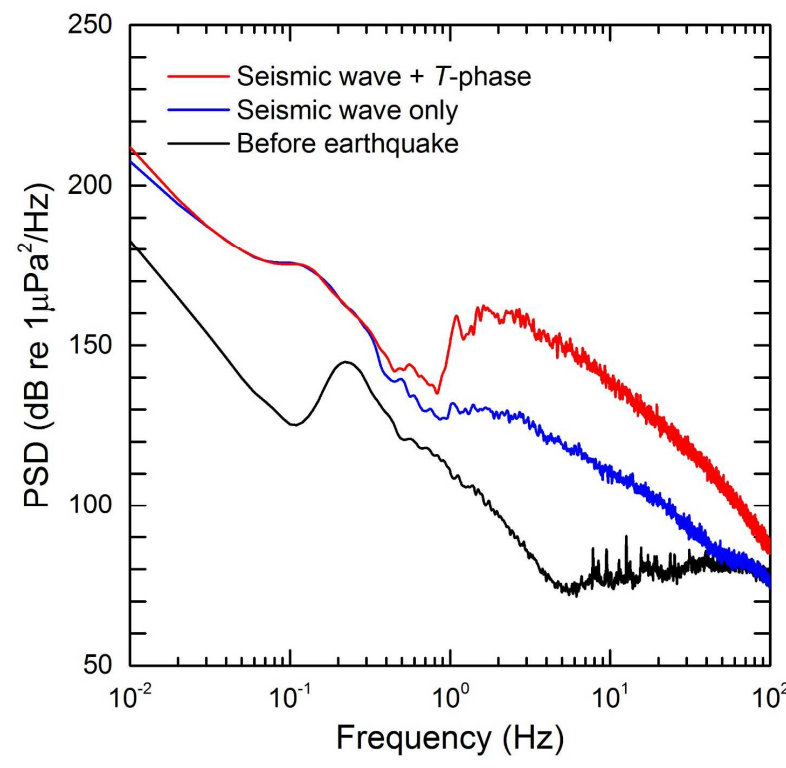

Fig.3 Power Spectral Density (PSD) of hydrophone recordings of seismic waves, $T$-phases and microseismic background noise observed at H11N1.

range in the presence of the low frequency seismic and microseismic noise typical of deep ocean locations ${ }^{8)}$.

\section{ANALYSIS OF T-PHASE SIGNALS}

\section{(1) Features of $\boldsymbol{T}$-phase signals}

Power Spectral Density (PSD) spectra of acquired pressure signals show clearly the presence of $T$-phase signals from the Tohoku earthquake. Figure 3 compares three spectra of $\mathrm{H} 11 \mathrm{~N} 1$ as a representative station: before the earthquake, after the seismic wave arrivals but before the $T$-phase arrivals, and after the $T$-phase arrivals.

The PSD before the earthquake is well comparable to ocean microseismic background noise reported by $\mathrm{Webb}^{8)}$. The PSD associated with the earthquake is a few tens of $\mathrm{dB}$ higher than the typical background noise. As for the PSD after the earthquake, two peaks at frequencies of $10^{-1} \mathrm{~Hz}$ and between 1 and $10 \mathrm{~Hz}$ are particularly visible. The presence of the $T$-phase signal increases further the PSD by several tens of $\mathrm{dB}$ in the frequency range between 1 and $10 \mathrm{~Hz}$. Below $1 \mathrm{~Hz}$, the seismic wave PSDs with and without the $T$-phase contribution are virtually the same.

\section{(2) Frequency-wavenumber analysis}

IMS hydroacoustic hydrophone stations are composed of hydrophone triplets as described above. With such an arrangement it is possible to process incident waves using a technique similar to seismic array analysis in order to evaluate the directivity of the $T$-phase. Some studies regarding array analysis in terms of $T$-phase signals using PMCC have been published in the past ${ }^{7,9)}$. The alternative presented here is based on frequency-wavenumber (F-K) analysis. In this technique, the averaged predominant frequency is calculated. A large number of horizontal bi-axial wavenumbers are then assumed as the starting point for the search for the most appropriate wavenumber, which is associated with the direction of arrival (and the corresponding time-domain inter-element delays) of the incoming signal. Using this method the back-azimuth and slowness of the incident signals are obtained. F-K analysis is one of the standard techniques in seismology ${ }^{10)}$, but it is not widely applied in ocean acoustics.

The main caveat in applying F-K analysis to hydroacoustic signals is that significant spatial aliasing is to be expected if the entire bandwidth of the hydrophones is used, since the distance between hydrophones in the triplet is large in comparison to the wavelength. To circumvent the problem, a three-step pre-processing of the data consisting of (i) high-pass filtering at $1 \mathrm{~Hz}$, (ii) envelope calculation using the Hilbert transform, and (iii) low-pass filtering of the envelope at $0.375 \mathrm{~Hz}$, is introduced. This makes it possible to obtain a low frequency signal envelope associated with the $T$-phase which does not give rise to spatial aliasing during the F-K analysis. The three steps can be explained as follows. In the first step, the $T$-phase signal is retrieved because the $T$-phase has a peak frequency above $1 \mathrm{~Hz}$ (see Figure 3). The envelope computed in the second step follows the variation of the $T$-phase signal. In the third step, low-pass filtering of the envelope waveform eliminates the short period variations but retains sufficient information to enable F-K analysis without spatial aliasing. Original $T$-phase signals and their final envelopes obtained by this technique are shown in Figure 4.

Through F-K analysis of these envelope signals the direction and slowness of the $T$-phase can be obtained (Figure 5). The present F-K analysis suggests that the incident wave originates from $325^{\circ}$ with $0.65 \mathrm{~s} / \mathrm{km}$ slowness, i.e., equivalent to $1.5 \mathrm{~km} / \mathrm{s}$ phase speed for the north triplet. This agrees with the theoretical value expected for a $T$-phase originating from the epicenter. For the south triplet the F-K analysis results in a less clear, but still unambiguous, estimation of the DOA. A possible explanation for this is the presence of Wake Island itself, which is near the direct path between the south triplet and the epicenter, while the north triplet has a free line-of-sight. Moreover, since H11S1 and H11S3 are aligned parallel to the expected $T$-phase wavefront 

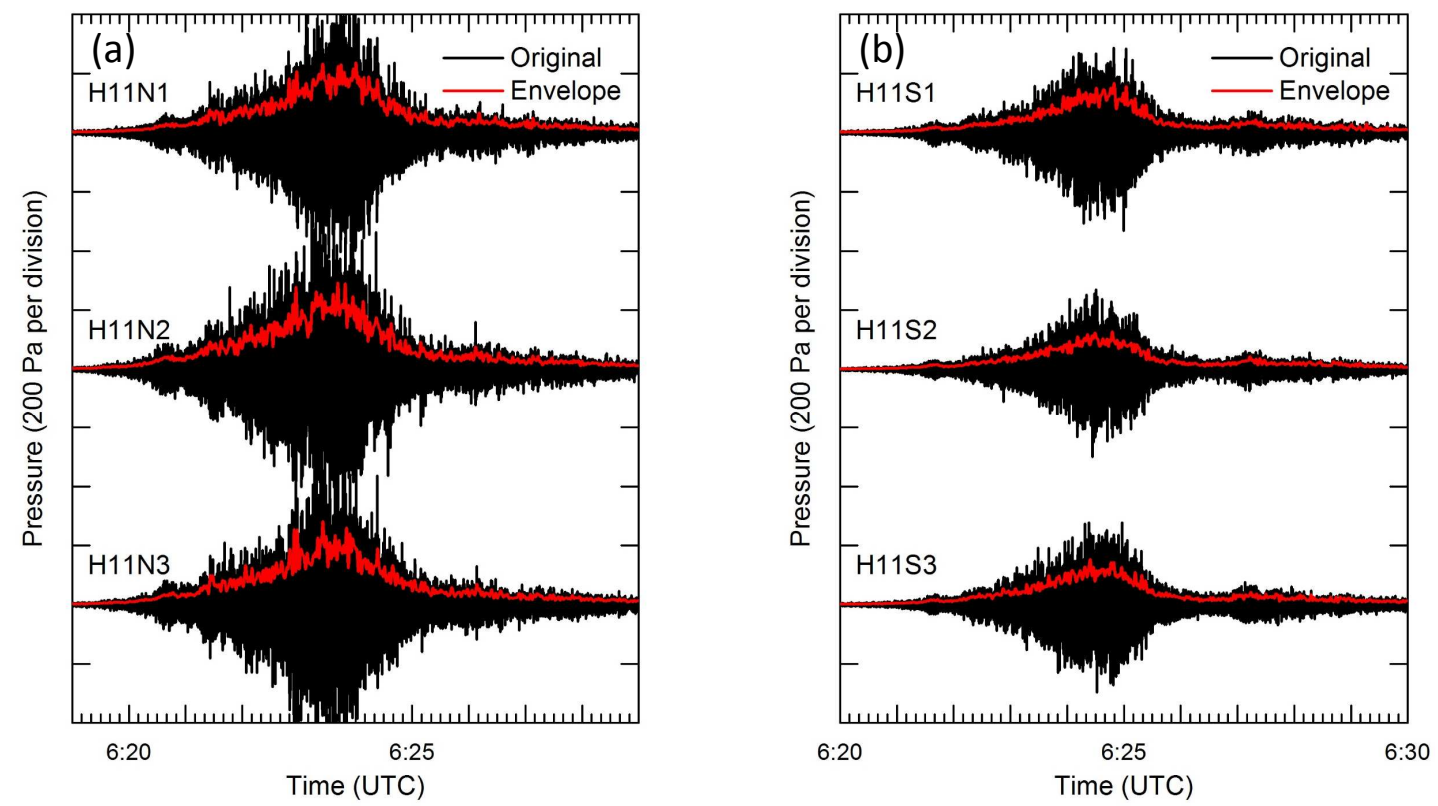

Fig.4 T-phase signals of HA11 hydrophone triplets (black lines) of (a) north and (b) south triplets and their envelopes after three-step processing (red lines).
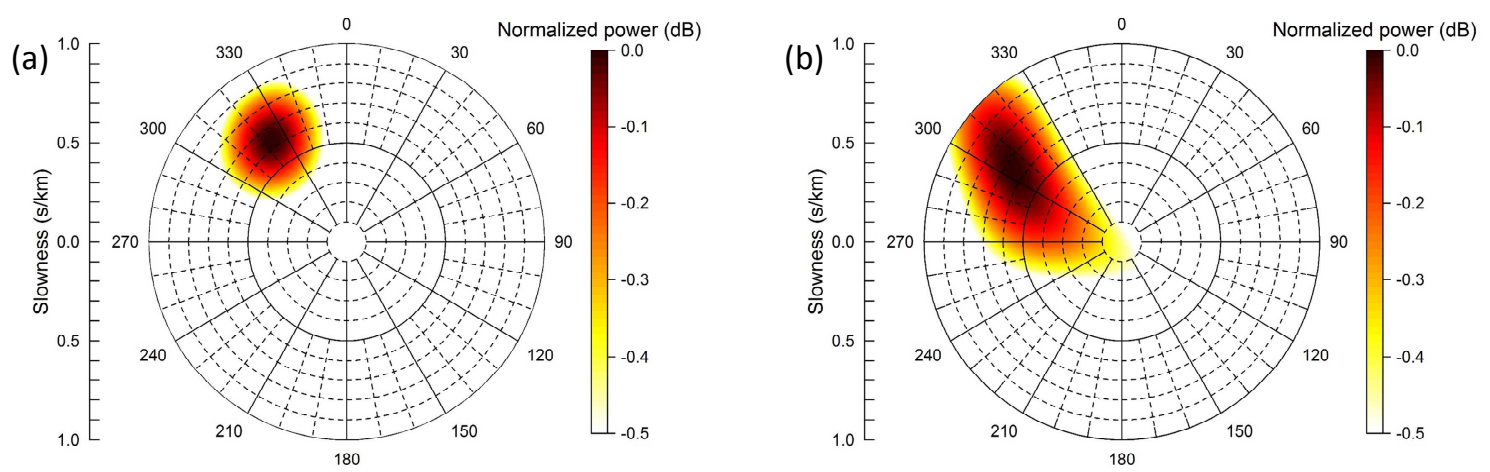

Fig.5 Back-azimuth and slowness mapping estimated from the F-K analysis from (a) north and (b) south triplets.

from the epicenter (see Figure 1), the resolution of the incident wave arrival angle from the NW direction can be expected to be lower than for the north triplet.

The results presented here show that the F-K analysis with envelope pre-processing can be used to estimate the direction and speed of the $T$-phase from oceanic earthquakes using IMS hydrophone triplets.

\section{ANALYSIS OF TSUNAMI SIGNALS}

\section{(1) Frequency response}

Measured frequency-amplitude-phase (FAP) responses of the HA11 hydrophones are available for the frequency band between $10^{-1} \mathrm{~Hz}$ and the upper limit of the sensor's frequency band. These are plotted as solid lines in Figure 6. The amplitude response is almost flat at frequencies above $10 \mathrm{~Hz}$, whereas it is attenuated gradually at frequencies below $10 \mathrm{~Hz}$. The amplitude drops steeply above 100
$\mathrm{Hz}$ because of the anti-aliasing filter of the hydrophones. However, for a very slow periodic phenomenon, such as a tsunami, the sensor response for much lower frequencies is required.

For the purpose of this work, it is therefore necessary to approximate the frequency response below $10^{-1} \mathrm{~Hz}$. This is possible by employing a response model that takes into account the amplitude and phase response of the filtering stages implemented in the hydrophone. In the case of the present realization of the IMS hydrophones, this is achieved with a three-pole transfer function model of the signal acquisition electronics, which takes into account three separate filter stages corresponding to the hydrophone capacitance, the signal conditioning circuitry, and a static pressure compensation orifice in the hydrophone enclosure. The modelled FAP responses are plotted as dashed lines in Figure 6. Although the model of the hydrophone response is based on an accurate description of each of the filter components, it is understood that some uncertainty is to be 


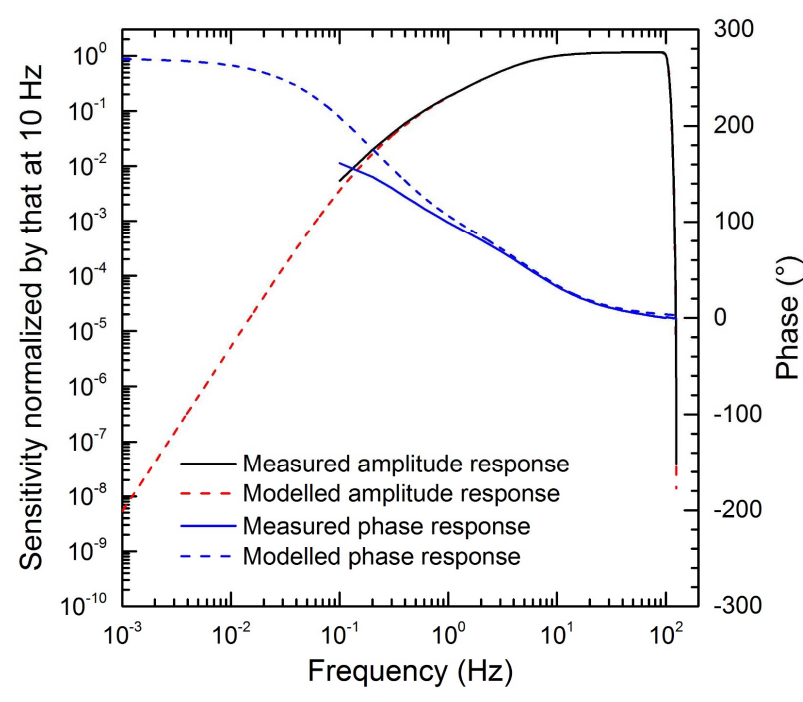

Fig.6 Frequency-amplitude and phase response of the HA11 hydrophones. Solid lines represent the measured calibrated response. Dashed lines are modelled using three-poles.

expected, especially at the lower band of the frequencies studied here. Instead, for the frequency band above $1 \mathrm{~Hz}$, the modelled filter response agrees well with the measured frequency response in terms of amplitude and phase.

\section{(2) Tsunami signals detected by hydrophones}

By using the modelled FAP responses, de-convolved waveforms are processed in the low frequency band down to $10^{-3} \mathrm{~Hz}$ (Figure 7). The de-convolved waveforms show low frequency signals which would otherwise be masked by the high frequency waveforms visible in the raw data. The de-convolved results are similar between the north and south triplets except for H11N2, which stands out with its larger amplitude. However, the signals recorded until 09:30 UTC are consistent between the three north hydrophones, showing that the hydrophones have consistent responses in this frequency band. This has been further substantiated by low-pass filtering the waveforms at $3 \times 10^{-3} \mathrm{~Hz}$ and verifying that the responses until 09:30 are consistent across the HA11 north hydrophones. The detailed investigation of the causes for the larger amplitude in the H11N2 recordings in the presence of the passing tsunami goes beyond the scope of this study and is the subject of on-going investigations.

The raw and the de-convolved waveforms and their spectrogram of H11N1 are shown in Figure 8. The spectrogram shows that from 06:00, the dispersive signal associated with the Rayleigh wave from the main shock of the Tohoku earthquake can be clearly identified in the frequency band between $10^{-2}$ and $10^{-1} \mathrm{~Hz}$. This activity related to the main shock can be seen to persist for up to approximately two

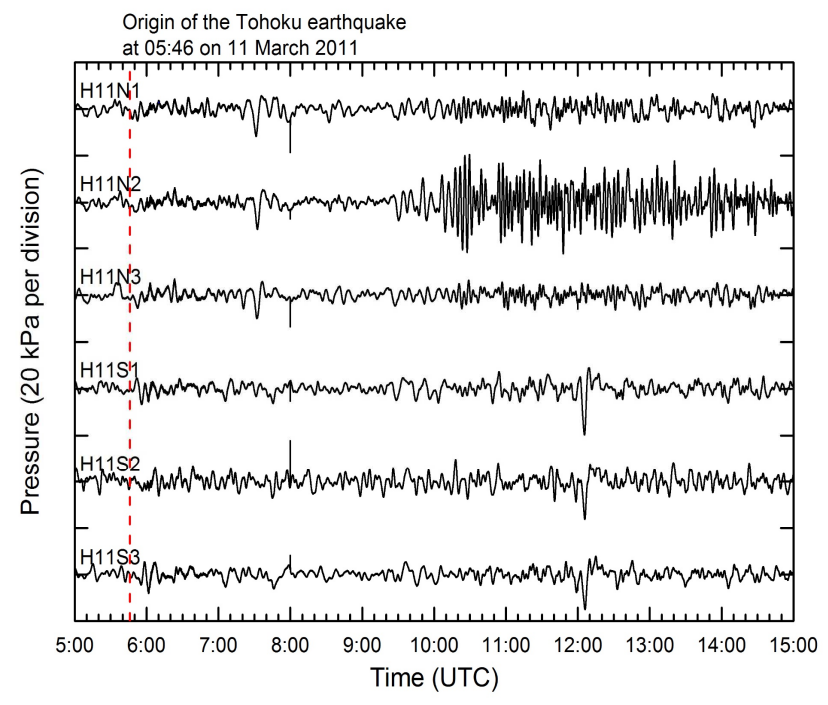

Fig.7 De-convolved pressure waveforms of HA11 hydrophone triplets using a model for the low frequency response of the sensor.

hours. Such long durations of activity are to be expected for a M9-class earthquake. Before the seismic wave dies out the $T$-phase can be seen arriving at 06:20, with predominant frequency content above $1 \mathrm{~Hz}$ and duration of approximately 10 minutes. Similar features have been confirmed in the time-frequency analysis of the other waveforms shown in Figure 7.

The de-convolution of the raw data puts into evidence the signals arriving at 09:30. The most notable feature is that this signal also indicates a dispersive wave, which is identified both in the raw and the de-convolved waveforms. This dispersive signal is consistent with the tsunami arriving at the station from the Tohoku earthquake. The travel time of approximately 3.5 to 4.0 hours after the earthquake is consistent with long period surface gravity wave theory. A calculated dispersion curve of a surface gravity wave, assuming an averaged water depth between the epicenter and HA11 of $5641 \mathrm{~m}$, is shown in Figure 8. The tsunami dispersion curve follows closely the low-frequency $\left(<10^{-2} \mathrm{~Hz}\right)$ dispersive feature which is visible in the spectrogram after 09:30 (expected tsunami arrival time). The distribution of peak frequencies and their variation is similar to those reported in a previous study of the 2004 Indian Ocean tsunami at $\mathrm{HA} 8^{5}$.

Although Figure 8 (b) shows that low frequency signals are evidenced by the de-convolution processing, their amplitude needs to be examined more quantitatively. The processing shows that it is possible to detect tsunami related signals with the IMS hydrophones. Other available offshore tsunami observations with bottom pressure gauges in the vicinity of the epicenter, such as the NOAA's Deep-ocean Assessment and Reporting of Tsunamis network, 

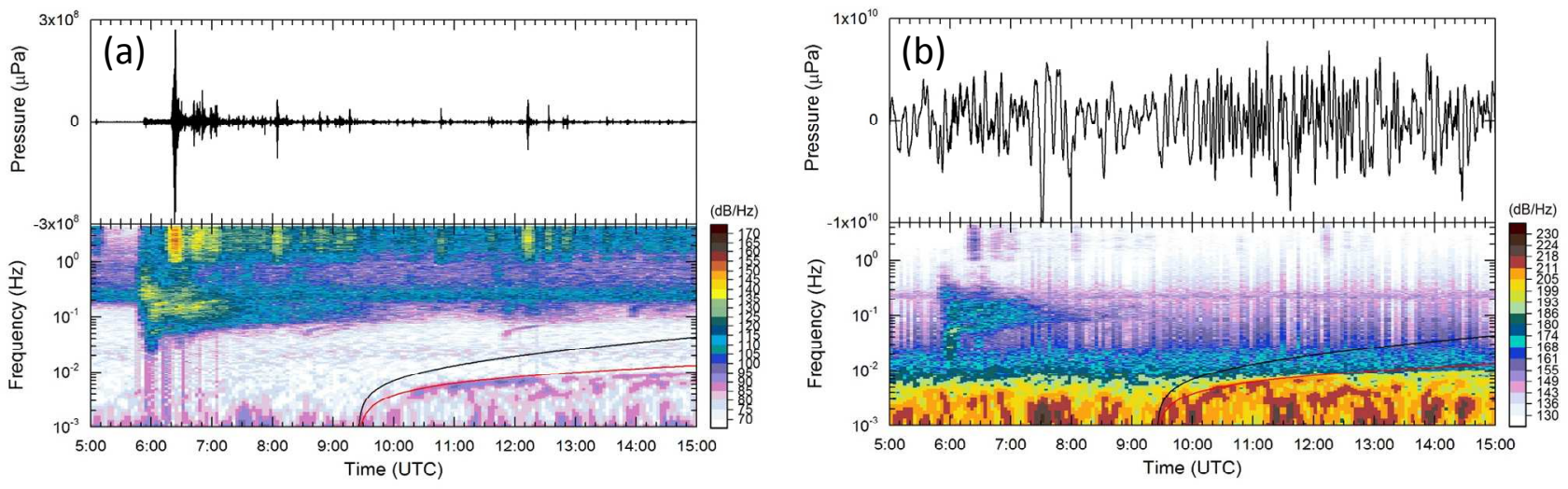

Fig.8 Waveforms and their spectrograms of (a) raw data and (b) de-convolved data of H11N1. Black and red lines in the spectrogram represent dispersion curves for channel and phase and group velocities of surface gravity waves with an averaged water depth of $5641 \mathrm{~m}$ between the epicenter and the HA11 hydroacoustic station.

show that the long period tsunami had amplitudes of a few tens of centimetres with a 30 minute period.

\section{CONCLUSION}

Raw pressure waveforms have been obtained by the IMS hydrophone triplets at Wake Island, which contain a wealth of information associated with the 2011 Tohoku earthquake. The data have been analyzed with the objective of studying both $T$-phase and tsunami signals from this mega-thrust earthquake.

The direction of the incident $T$-phase signal has been estimated using F-K analysis with an envelope pre-processing technique, which makes it possible to circumvent the spatial aliasing otherwise caused by the large inter-element separation. This has shown that the direction of $T$-phase signals detected by the IMS water-column hydrophone triplets can be estimated for both triplets.

To study the tsunami related signals de-convolved data are processed by using a three-pole frequency-amplitude-phase model of the hydrophone response. This makes it possible to represent the signal features down to $10^{-3} \mathrm{~Hz}$ which are associated with the pressure disturbance caused by the tsunami propagating over the hydrophone. The observed features are consistent with expected tsunami arrival times and low-frequency signal dispersion up to $10^{-2} \mathrm{~Hz}$.

This study shows that the IMS hydrophone triplets are potentially useful for the detection of both $T$-phase and tsunami signals. However, by design, the IMS hydrophones are not sufficiently sensitive in the frequency band below $10^{-3} \mathrm{~Hz}$ to enable the study of long period tsunami signals using the method presented here. To study such long period signals, which would be more similar to those recorded by traditional pressure gauges, other alternative techniques may be needed.
ACKNOWLEDGMENT: We thank Dr. Nurcan Meral Ozel, CTBTO/IMS Director, Dr. Tryggvi Edwald, Dr. Ronan Le Bras and Dr. David Brown (CTBTO/IDC) for their scientific contribution. The SEIZMO software in MATLAB was used in the present F-K analysis. This study was part of the CTBTO's Cost Free Expert program supported by the Ministry of Foreign Affairs of Japan.

\section{REFERENCES}

1) Ewing, M., Tolstoy, I. and Press, F. : Proposed use of the T phase in tsunami warning systems, Bull. Seism. Soc. Am., Vol. 40, pp. 53-58, 1950.

2) Walker, D. A. and Bernard, E. N. : Comparison of $T$-phase spectra and tsunami amplitude for tsunamigenic and other earthquakes, J. Geophys. Res., Vol. 98, No. C7, pp. 12557-12565, 1993.

3) Okal, E. : The generation of $T$ waves by earthquakes, $A d$ vances Goephys., Vol. 49, Chap. 1, pp. 1-65, 2008.

4) Hanatani, H., Koshimura, S. and Meguro. K. : A study on detecting T-phase for tsunami warning, J. JSCE B2 Coastal. Eng., Vol. 66, No. 1, pp. 1321-1325, 2010. (in Japanese)

5) Hanson, J.A., and Bowman, J.R., Dispersive and reflected tsunami signals from the 2004 Indian Ocean tsunami observed on hydrophones and seismic stations, Geophys. Res. Lett., Vol. 32, L17606, doi:10.1029/2005GL023783, 2005.

6) Okal, E., Talandier, J. and Reymond, D. : Quantification of hydrophone records of the 2004 Sumatra tsunami, Pure Appl. Geophys., Vol. 164, pp. 309-323, 2007.

7) Guilbert, J., Vergoz, J., Schisselé, E., Roueff, A. and Cansi, Y. : Use of hydroacoustic and seismic arrays to observe rupture propagation and source extent of the $M_{w}=9.0 \mathrm{Su}$ matra earthquake, Geophys. Res. Lett., Vol. 32, L15310, doi:10.1029/2005GL022966, 2005.

8) Webb S. C. : Broadband seismology and noise under the ocean, Rev. Geophys., Vol. 36, pp. 105-142, 1998.

9) Yun, S. and Lee, W. S. : Hydroacoustic observation on the 2011 Tohoku earthquake, Jigu-Mulli-wa-Mulli-Tamsa, Vol. 16, No. 4, pp. 234-239, 2013.

10) Capon, J. : High-resolution frequency-wavenumber spectrum analysis, Proc. IEEE, Vol. 57, No. 8, pp. 1408-1418, 1969.

(Received March 16, 2016) 